\title{
Resultados preliminares del tratamiento con luz pulsada intensa regulada en el síndrome de ojo seco
}

\section{Preliminary results of treatment with Intense Regulated Pulsed Light in Dry Eye Syndrome}

\author{
Giuseppe Miotto-Montesinos ${ }^{1,2}$, Eduardo Rojas-Alvarez ${ }^{2,3 *}$ y Diego M. Torres-Piedra² \\ ${ }^{1}$ Facultad de Medicina, Universidad del Azuay; ${ }^{2}$ Centro Oftalmológico Exiláser; ${ }^{3}$ Facultad de Medicina, Universidad de Cuenca. Cuenca, Ecuador
}

\section{Resumen}

Objetivo: Describir los resultados preliminares del empleo de luz pulsada intensa regulada (IRPL) en pacientes con síndrome de ojo seco por disfunción de las glándulas de Meibomio (DGM). Método: Se realizó un estudio descriptivo, longitudinal y prospectivo en 32 pacientes con diagnóstico de síndrome de ojo seco, en los cuales se aplicó tratamiento con IRPL, durante el periodo de abril a diciembre de 2017, en el Centro Oftalmológico Exiláser, Cuenca, Ecuador. Se realizaron 3 sesiones de tratamiento: día 0, día 15 y día 45. Se aplicaron frecuencias absolutas, relativas, prueba de Pearson y test de Fischer. Resultados: Después del tratamiento con IRPL, en el 87.5\% de los casos se constató un tiempo de ruptura de la película lagrimal (BUT) superior a $10 \mathrm{~s}$, con dependencia entre aplicación del tratamiento e incremento del BUT. Se obtuvo una disminución de los signos encontrados en el examen del borde palpebral, que fue más notoria en la blefaritis. En el $81.3 \%$ de los casos fue suficiente una presión suave al realizar la expresión glandular para obtener características de líquido claro transparente en el $62.5 \%$ de los casos. Se obtuvo mejoría en todos los indicadores del test de OSDI (Ocular Surface Disease Index). Conclusiones: Los resultados presentados muestran una mejora tanto en los signos clínicos y síntomas del síndrome de ojo seco por DGM, después de un curso de tres sesiones de IRPL, durante un período de 45 días, no obstante, se requieren estudios futuros a largo plazo relacionados con este tratamiento.

Palabras clave: Luz pulsada intensa. Ojo seco. Glándulas de Meibomio.

\section{Abstract}

Objective: To describe the preliminary results of the use of Intense Regulated Pulsed Light (IRPL) in patients with Dry Eye Syndrome due to Meibomian Gland Dysfunction (MGD). Methods: A descriptive, longitudinal and prospective study was performed in 32 patients diagnosed with Dry Eye Syndrome, in which treatment with IRPL was applied between April-December 2017 at the Ophthalmological Center Exilaser, in Cuenca, Ecuador. Three treatment sessions were performed on Day 0, Day 15 and Day 45. Absolute and relative frequencies, Pearson test and Fischer test were used for statistical analyses. Results: After treatment with IRPL, tear film breakup time (BUT) greater than 10 seconds was observed in $87.5 \%$ of the patients, with a correlation between treatment application and a BUT greater than 10 seconds. There was a decrease in the signs observed in the eyelid margin; this decrease was more evident in blepharitis cases. In $81.3 \%$ of the cases, a gentle

Correspondencia:

${ }^{*}$ Eduardo Rojas-Alvarez

Federico Proaño 4-26 y Remigio Crespo Fecha de recepción: 02-02-2018

Cuenca, Ecuador

Fecha de aceptación: 19-09-2018

Disponible en internet: 01-11-2018

E-mail: drerojasalvarez@gmail.com

DOI: 10.24875/RMO.M18000051

Rev Mex Oftalmol. 2018;92(6):292-299 (http://creativecommons.org/licenses/by-nc-nd/4.0/). 
pressure was enough when performing the glandular expression, in order to obtain a clear liquid in $62.5 \%$ of the cases. All the OSDI test items showed improvement. Conclusions: Our results show an improvement of the clinical signs and symptoms of Dry Eye Syndrome due to MGD after a course of three IRPL sessions over a 45-day period. However, more IRPL studies with a longer follow-up are warranted.

Key words: Intense Pulsed Light Therapy. Dry eye. Meibomian gland.

\section{Introducción}

El síndrome de ojo seco es uno de los motivos de consulta más frecuentes en Oftalmología, y afecta la calidad de vida de cientos de miles de personas en el mundo ${ }^{1}$. Cada día son más los pacientes que acuden al especialista para tratar de atenuar los síntomas tan molestos que se producen, y buscar posibles causas y soluciones a su malestar. En la mayoría de las ocasiones se observa coexistencia entre la presencia de ojo seco y otras entidades nosológicas de la superficie ocular, lo cual hace complejo, aún más, el manejo y resolución de estos casos ${ }^{2}$.

La recurrencia y elevada frecuencia de los síntomas obliga a estos pacientes al uso continuo de sustitutos lagrimales e, incluso, a variar cada cierto tiempo el medicamento usado, en busca de nuevas alternativas para su enfermedad, ya sea indicado por el galeno 0 por decisión del propio paciente.

A lo largo del desarrollo tecnológico de la especialidad, se han realizado varios intentos por mitigar esta sintomatología y de alguna forma "curar» un cuadro clínico crónico que en no pocas ocasiones se presenta ante los oftalmólogos como el paciente al que «no queremos atender» o que "ya no tiene solución».

Dentro de estas opciones de tratamiento, para el síndrome de ojo seco, causado por disfunción de las glándulas de Meibomio (DGM) se encuentra el sistema IPL (Intense Pulsed Light), que es una fuente de luz de alta intensidad que emite luz policromática y no coherente en un amplio espectro ${ }^{3}$.

Los filtros únicos en la luz pulsada intensa regulada (IRPL) limitan las longitudes de onda entre $550 \mathrm{~nm}$ y $1200 \mathrm{~nm}$ para tratamientos de ojo seco, específico para tratar solo la DGM, que es la causa más común del síndrome de ojo seco. La luz se dirige al tejido de la piel y luego es absorbida por la estructura objetivo, lo que da como resultado la producción de calor (> $80^{\circ} \mathrm{C}$ ), que destruye las lesiones cutáneas pigmentadas. Se pueden seleccionar longitudes de onda apropiadas para objetivos diferentes dependiendo del comportamiento de absorción y de la profundidad de penetración de la luz emitida, y se pueden elegir filtros específicos para limitar la entrega de longitudes de onda a la zona de tratamiento, dando como resultado un suministro térmico selectivo ${ }^{4,5}$.

El dispositivo de IRPL de tercera generación está diseñado específicamente para aplicaciones perioculares con múltiples pulsos de luz esculpidos homogéneamente (E-Eye, E-Swin, París, Francia), es el único dispositivo de IRPL certificado médicamente para tratar la $\mathrm{DGM}^{4-6}$. Dentro de sus parámetros fundamentales incluye un área a tratar de $7.5 \mathrm{~cm}^{2}$ con una longitud de onda entre 580 y $1200 \mathrm{~nm}$, en temperaturas que oscilan desde $-5^{\circ} \mathrm{C} \mathrm{a}+65^{\circ} \mathrm{C}$.

Con el advenimiento de esta novedosa tecnología a nuestra institución, hemos comenzado a emplearla en pacientes con síndrome de ojo seco por DGM.

\section{Objetivo}

Describir los resultados preliminares del empleo de IRPL en pacientes con síndrome de ojo seco por disfunción de glándulas de Meibomio.

\section{Método}

Se realizó un estudio descriptivo, longitudinal y prospectivo en 32 pacientes, con diagnóstico de síndrome de ojo seco, en los cuales se aplicó el tratamiento con IRPL, en el periodo de abril a diciembre de 2017, en el Centro Oftalmológico Exiláser, Cuenca, Ecuador. Se realizaron 3 sesiones de tratamiento: día 0, día 15 y día 45 .

\section{Criterios de selección de los casos}

Criterios de inclusión:

- Pacientes con síndrome de ojo seco por disfunción de las glándulas de Meibomio.

- Edad: mayores de 18 años.

- Motivación: por uso menos frecuente de sustitutos lagrimales.

Criterios de exclusión:

- Color de piel negro (grado VI de piel).

- Uso de medicación que incrementa la fotosensibilidad.

- Diabetes mellitus no compensada. 


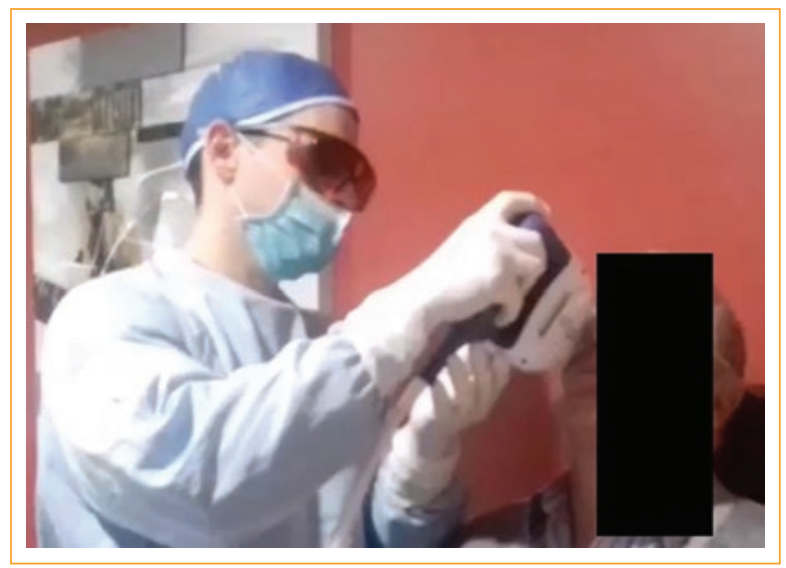

Figura 1. Aplicación de tratamiento con IRPL.

- Lesiones de piel activas en la zona de tratamiento.

- Embarazo.

- Lesiones de la piel por quemadura solar un mes previo al tratamiento.

- Historia previa de alergia al sol.

- Hemofilia, porfiria cutánea tardía.

- Marcapasos, desfibrilador.

- Epilepsia.

\section{Protocolo de tratamiento}

- Limpieza y desinfección de la región de la piel inferior y lateral al globo ocular.

- Cierre de ambos ojos y colocación de un oclusor bilateral.

- Colocación de gel (de conducción) en la zona de tratamiento, desde la piel de la región temporal orbitaria, piel inferior a los párpados inferiores, incluida la región de la nariz, hasta la región contralateral.

- Aplicación de 5 flash, comenzando en la región nasal interna hasta la región temporal, en ambos ojos (Fig. 1).

- Nivel de energía utilizada: $12.2 \mathrm{~J} / \mathrm{cm}^{2}$

- Remoción de gel de la región de la cara e instilación de lidocaína (colirio) (Alcon) 1 gota en ambos ojos.

- Examen en biomicroscopio anterior y realización de expresión de la glándulas de Meibomio, previa aplicación de anestesia tópica, con aplicador estéril (cotonete), situado en la conjuntiva palpebral en el área de la glándula de Meibomio, mientras se aplica presión en la piel con el dedo índice próximo a la misma glándula a explorar, por 30 segundos.

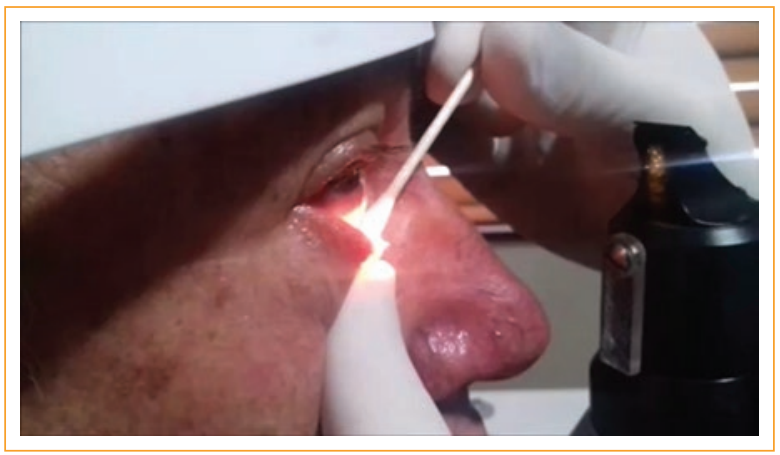

Figura 2. Expresión glandular posterior al tratamiento con IRPL.

- El proceder se repite en ambos ojos, en los párpados superior e inferior. El paciente debe mirar en dirección contraria al párpado que se examina (Fig. 2).

- Limpieza de los bordes palpebrales, específicamente de la secreción meibomiana obtenida.

- Posprocedimiento:

- Cristaltears ${ }^{\circledR}$ (colirio) (carboximetilcelulosa sódica $0.5 \%$ ): 1 gota cada $4 \mathrm{~h}$.

- Poenbiotic $^{\circledR}$ (colirio) (ciprofloxacino 0.3\%, dexametasona $0.1 \%$.): 1 gota cada $4 \mathrm{~h}$, hasta 5 días después de la sesión.

- Ciprodex $^{\circledR}$ (ungüento) (ciprofloxacino $0.3 \mathrm{~g}$, dexametasona $0.1 \mathrm{~g}$ ): aplicar a las 10:00 pm, hasta 5 días después de la sesión.

\section{Características generales de la tecnología}

Se utilizó la tecnología E-Eye (E-Swin, Francia), comercializado en Ecuador por SERVICESA (Fig. 3).

Tecnología: IRPL

Área tratada: $7.5 \mathrm{~cm}^{2}$

Dimensiones: 345 x 320 × 440 mm

Peso: $11.5 \mathrm{Kg}$

Voltaje: $100-240 \mathrm{~V}$

Temperatura: $-5^{\circ} \mathrm{C} \mathrm{a}+65^{\circ} \mathrm{C}$

Certificación: Medical CE, TGA (Australia), WAND (New-Zealand)

Condición que trata: DGM

Longitud de onda: $580-1200 \mathrm{~nm}$

\section{Variables del estudio}

Las siguientes variables fueron evaluadas en 2 momentos: el día 0 (previo al primer tratamiento) y el día 60 (15 días después de la tercera sesión): 

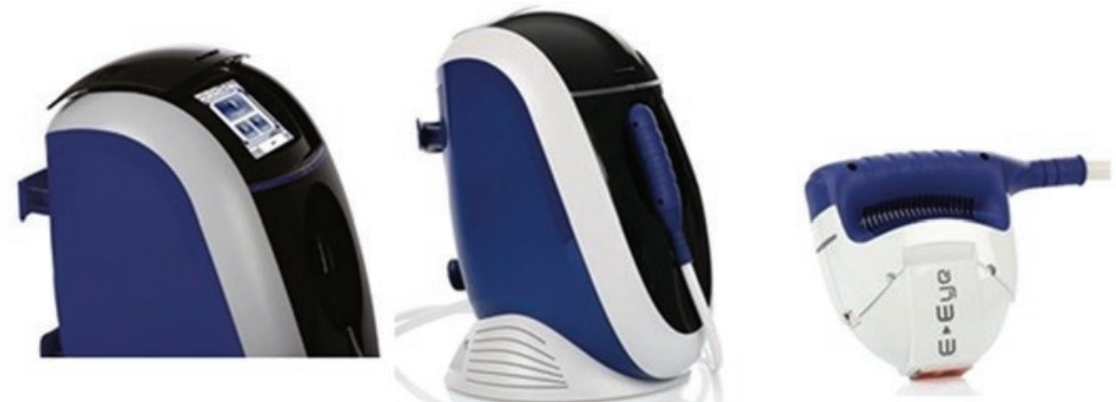

Figura 3. Tecnología E-Eye con IRPL para el tratamiento del síndrome de ojo seco por DGM.

- Tiempo de ruptura de la película lagrimal (BUT). Se realizó en biomicroscopio anterior con fluoresceína y luz azul cobalto, expresado en segundos en la escala siguiente: más de $10 \mathrm{~s}$, entre 5 y $10 \mathrm{~s}$, y menos de $5 \mathrm{~s}$.

- Signos en el borde libre del párpado. Fueron evaluados la presencia de irregularidad, telangectasias, blefaritis, triquiasis.

- Características de la expresión glandular. Evaluada en biomicroscopio anterior, expresada de acuerdo a la siguiente escala: líquido claro transparente, líquido no transparente, granular (grumos) y sólido (toothpaste).

- Presión realizada para la expresión glandular. Presión que realiza el médico en el momento de la expresión glandular, expresada según la escala siguiente: suave, moderada, severa.

- OSDI (Ocular Surface Disease Index). Cuestionario que incluye 3 grupos de preguntas relacionadas con la frecuencia de aparición de determinados eventos (síntomas, actividades cotidianas y situaciones específicas), donde el paciente responde en una escala del 0 al 4 con las categorías siguientes: 0 , en ningún momento; 1 , casi en ningún momento; 2 , el $50 \%$ del tiempo; 3 , casi en todo el tiempo; 4, Todo el tiempo. El cuestionario se realizó de forma individual, por un profesional no relacionado con la aplicación del tratamiento. Se obtuvo el valor de la media de la muestra, obtenida en cada categoría por paciente.

\section{Análisis estadístico}

Los cálculos obtenidos por variable fueron incorporados a una tabla de datos en SPSS ${ }^{\circledR}$ versión 21.0. Se utilizaron métodos de estadística descriptiva e
Tabla 1. Tiempo ruptura de la película lagrimal

\begin{tabular}{|l|c|c|c|c|}
\hline \multirow{2}{*}{ Tiempos } & \multicolumn{2}{|c|}{ Antes } & \multicolumn{2}{c|}{ Después } \\
\cline { 2 - 5 } & $N^{0}$ & Porcentaje & $N^{0}$ & Porcentaje \\
\hline Mayor de $10 \mathrm{~s}$ & 2 & 3.1 & 56 & 87.5 \\
\hline Entre 5 y $10 \mathrm{~s}$ & 48 & 75 & 6 & 9.4 \\
\hline Menor de $5 \mathrm{~s}$ & 14 & 21.9 & 2 & 3.1 \\
\hline Total & 64 & 100 & 64 & 100 \\
\hline
\end{tabular}

inferencial expresados en frecuencias absolutas y frecuencias relativas. Se aplicó la prueba chi cuadrado de Pearson para una tabla de contingencia con el objetivo de verificar la posible dependencia de la aplicación del tratamiento (antes y después del tratamiento) en el BUT. Se procedió posteriormente a un análisis de los residuos estandarizados para la verificación de cuáles de las relaciones crean esta dependencia. Para la variable características de expresión glandular se aplicó el test exacto de Fisher. Se utilizó un nivel de significación del 95\%. Los resultados fueron presentados en tablas y gráficos. Los pacientes otorgaron su consentimiento para el tratamiento y su participación en el estudio.

Adjunto Anexo del Comité de Ética Asistencial de Salud: Bioética Exiláser. Autorización - Código: 201803-03. Acuerdo 3 del 2018.

\section{Resultados}

Como se observa en la tabla 1, antes del procedimiento, la mayoría de los pacientes tenían un BUT entre 5 y $10 \mathrm{~s}$, posterior al IRPL se obtuvo una mayoría de pacientes con más de $10 \mathrm{~s}$ de BUT. 
Los resultados de la prueba chi cuadrado de Pearson sugieren dependencia entre la aplicación de IRPL y el BUT $(p=0.000<\alpha=0.05$ ). El análisis de los residuos estandarizados refleja que en cada una de las intersecciones hay diferencias significativas a un $1 \%$ de nivel de significancia (Residuos corregidos > 2.57), y valorando el signo se ha podido detectar que existe un incremento significativo, después del tratamiento, entre los BUT mayor de $10 \mathrm{~s}$ y disminuciones significativas entre los 5 y $10 \mathrm{~s}$ y menos de $5 \mathrm{~s}$.

En cuanto a los signos observados en el borde libre del párpado, la blefaritis tuvo un franco predominio antes del tratamiento, acompañada en orden de frecuencia de la irregularidad del borde palpebral, las telangectasias y triquiasis. Después de IRPL hubo una disminución en la presencia de blefaritis y triquiasis, menos perceptible esta disminución en la presencia de irregularidades del borde y telangectasias, que se mantuvieron con valores muy similares a los obtenidos previo al tratamiento (tabla 2).

La expresión glandular en forma de grumos predominó antes del tratamiento, seguida de la expresión en forma de líquido no transparente. Posterior a IRPL, más de la mitad de los pacientes tenían expresión glandular líquida transparente, aunque se mantienen casos, en menor medida, con expresión granular.

La realización de un test exacto de Fisher refleja dependencia de las variables categóricas tratadas $(p=0.000<$ $\alpha=0.05$ ). Un análisis de los residuos corregidos sugiere que esta dependencia está dada por la influencia positiva del incremento altamente significativo $(\alpha=0.01)$ de casos con presencia de líquido claro transparente después de aplicado el tratamiento y la disminución significativa ( $\alpha=$ 0.01 ) de casos con líquido no transparente y granular, grumos. No se observa diferencia significativa entre antes y después del tratamiento en el caso de característica sólido de expresión glandular $(\alpha=0.05)$ (tabla 3).

Se obtuvo un predominio de presión moderada, realizada para la expresión glandular. Antes del tratamiento fue moderada en la mayoría de los ojos, posterior a IRPL una suave presión fue necesaria en la gran mayoría de los ojos (tabla 4).

En cuanto a los resultados del test OSDI, se obtuvo disminución de la puntuación referida por los pacientes, en las 3 esferas que incluye el test, después de aplicado el tratamiento, con predominio de sensación de arenilla, dificultad para conducir por la noche y molestias ante la exposición al viento (Figs. 4, 5 y 6)

\section{Discusión}

La mejoría subjetiva de los pacientes estudiados, obtenida por test de OSDI, manifiesta una disminución de
Tabla 2. Signos al examen del borde libre del párpado

\begin{tabular}{|l|c|c|c|c|}
\hline \multirow{2}{*}{ Signos } & \multicolumn{2}{|c|}{ Antes } & \multicolumn{2}{c|}{ Después } \\
\cline { 2 - 5 } & $N^{0}$ & Porcentaje & $N^{0}$ & Porcentaje \\
\hline Irregularidad & 28 & 43.8 & 24 & 37.5 \\
\hline Telangectasias & 21 & 32.8 & 20 & 31.3 \\
\hline Blefaritis & 47 & 73.4 & 12 & 18.8 \\
\hline Triquiasis & 13 & 20.3 & 5 & 7.8 \\
\hline
\end{tabular}

Tabla 3. Características de la expresión glandular

\begin{tabular}{|l|c|c|c|c|}
\hline \multirow{2}{*}{ Características } & \multicolumn{2}{|c|}{ Antes } & \multicolumn{2}{c|}{ Después } \\
\cline { 2 - 5 } & $N^{0}$ & Porcentaje & $N^{0}$ & Porcentaje \\
\hline $\begin{array}{l}\text { Líquido claro } \\
\text { transparente }\end{array}$ & 2 & 3.1 & 40 & 62.5 \\
\hline $\begin{array}{l}\text { Líquido no } \\
\text { transparente }\end{array}$ & 21 & 32.8 & 8 & 12.5 \\
\hline $\begin{array}{l}\text { Granular, grumos } \\
\text { Sólido }\end{array}$ & 38 & 59.4 & 14 & 21.9 \\
\hline Total & 3 & 4.7 & 2 & 3.1 \\
\hline
\end{tabular}

Tabla 4. Presión realizada para la expresión glandular

\begin{tabular}{|l|c|c|c|c|}
\hline \multirow{2}{*}{ Presión } & \multicolumn{2}{|c|}{ Antes } & \multicolumn{2}{c|}{ Después } \\
\cline { 2 - 5 } & $N^{0}$ & Porcentaje & $\mathbb{N}^{0}$ & Porcentaje \\
\hline Suave & 16 & 25 & 52 & 81.3 \\
\hline Moderada & 42 & 65.6 & 10 & 15.6 \\
\hline Severa & 6 & 9.4 & 2 & 3.1 \\
\hline Total & 64 & 100 & 64 & 100 \\
\hline
\end{tabular}

Ios síntomas por DGM, que es una causa común del ojo seco evaporativo, que afecta a casi el $70 \%$ de la población en algunas partes del mundo ${ }^{1}$. Se manifiesta con los síntomas de la superficie ocular: ardor, irritación, agudeza visual fluctuante, ojo rojo, entre otros ${ }^{7}$ (Fig. 7).

Es frecuente que estos síntomas, combinados con opciones de tratamiento ineficaces, afecten la calidad de vida de los pacientes. En la DGM, las glándulas pueden estrecharse, existe atrofia de los acinos e hiperqueratinización ${ }^{8}$. El meibum aumenta en viscosidad, lo que fomenta la proliferación de bacterias comensales. Estas bacterias secretan lipasas que pueden alterar la composición de los lípidos en el meibum, y aumentar el nivel de colesterol esterificado (y su punto de fusión), lo que reduce aún más la producción glandular ${ }^{9-11}$. 


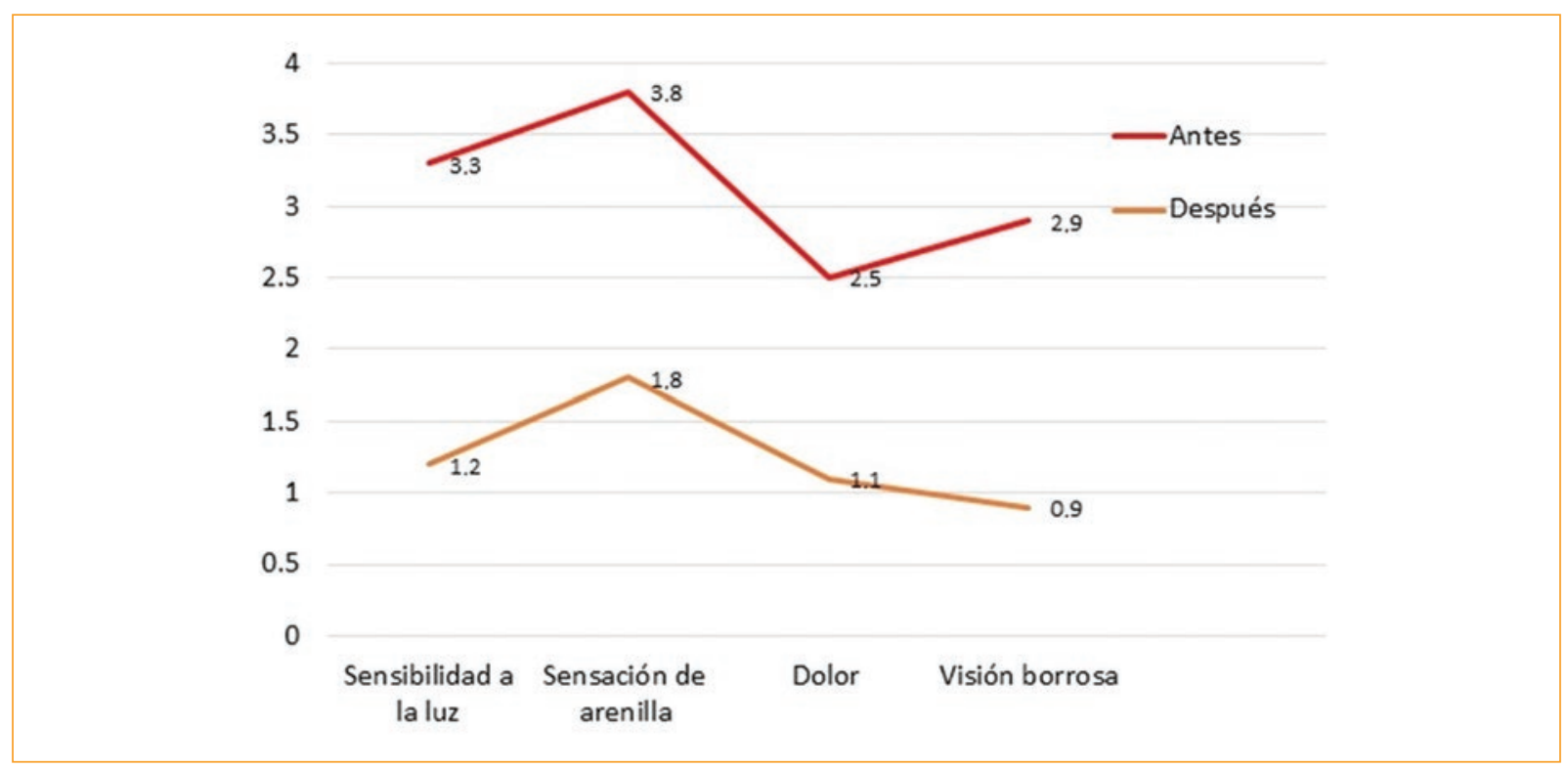

Figura 4. Test OSDI: Síntomas (antes y después de IRPL).

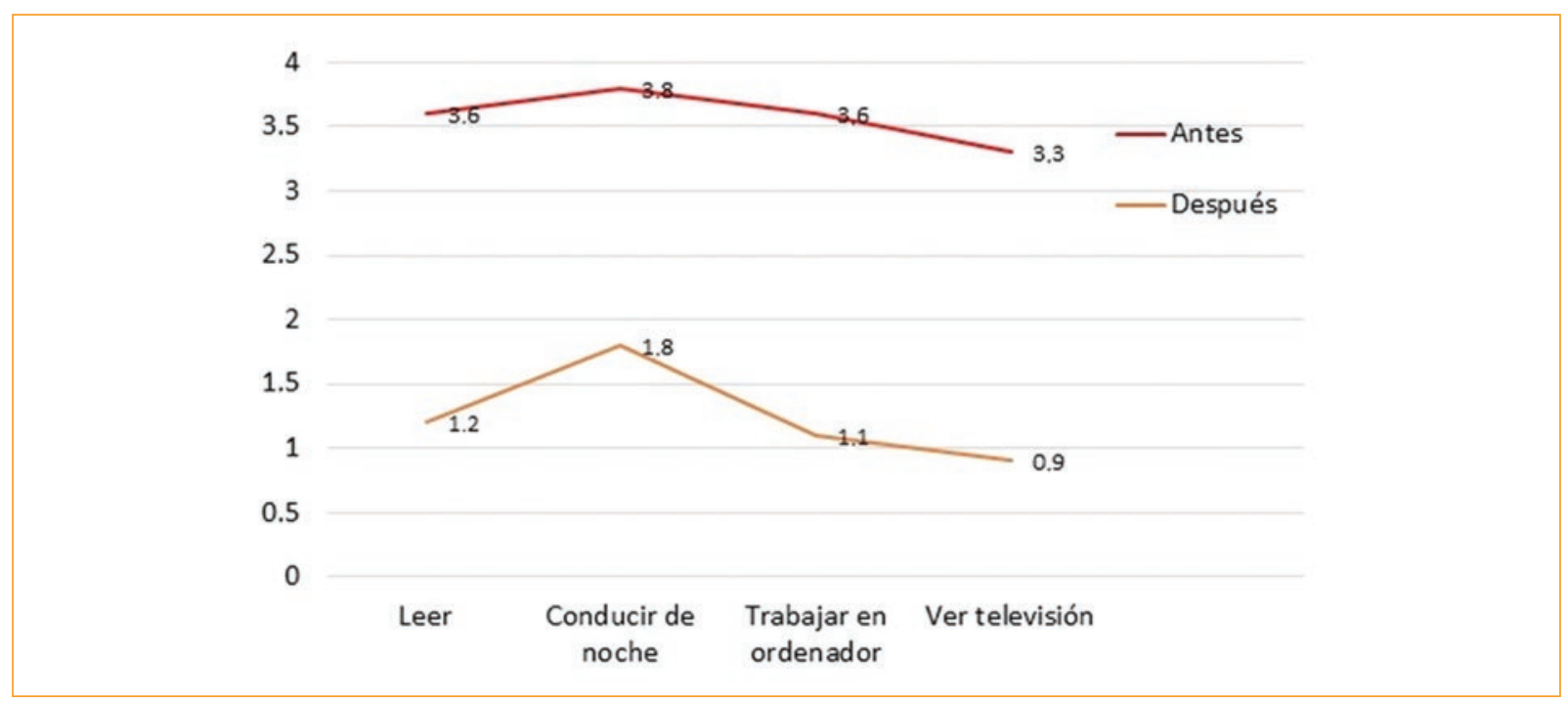

Figura 5. Test OSDI: Actividades cotidianas (antes y después de IRPL).

Los signos biomicroscópicos de la DGM pueden no ser notorios en el caso de los cuadros obstructivos glandulares y, en otros casos, pueden incluir orificios de las glándulas de Meibomio obstruidos o tapados, junto con el engrosamiento del margen palpebral, irregularidad, telangectasia e hiperemia del mismo ${ }^{12,13}$.

En los pacientes estudiados se observó la solidificación de las secreciones en la expresión de las glándulas en los casos más graves. El tiempo de ruptura de la lágrima se reduce con más frecuencia y la película lagrimal frecuentemente está contaminada por restos endógenos y espuma ${ }^{11-13}$. En última instancia, las glándulas de Meibomio no secretan una suficiente capa de aceite para la película lagrimal, lo que permite una mayor tasa de evaporación de la capa acuosa subyacente, que a su vez conduce a síntomas de ojo seco e inflamación de la superficie ocular ${ }^{14,15}$.

El mecanismo por el cual en la DGM los signos y síntomas mejoran después del tratamiento con IRPL sigue siendo un tema controversial y existen diferentes hipótesis que lo sustentan. Los mecanismos propuestos incluyen la transferencia de calor, lo que suaviza la 


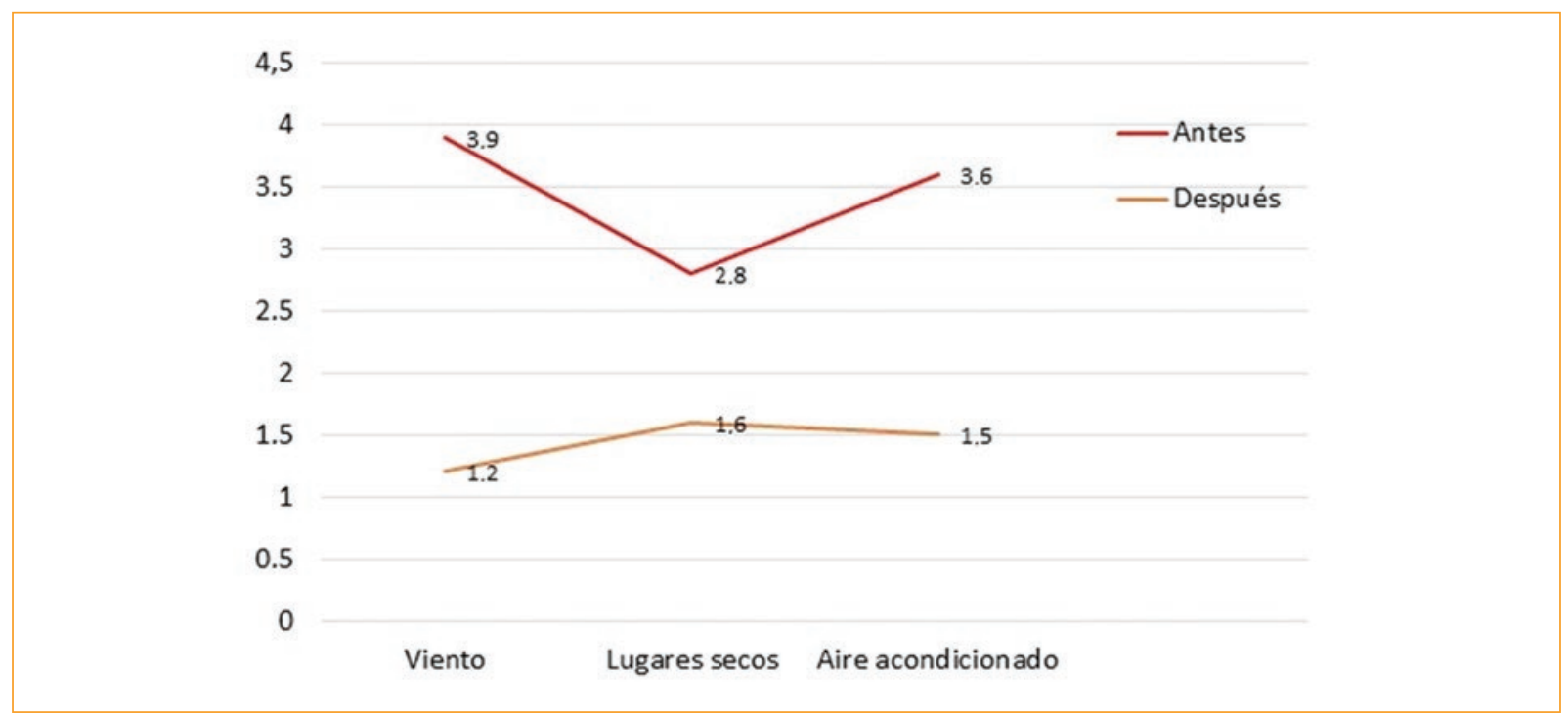

Figura 6. Test OSDI: Situaciones (antes y después de IRPL).

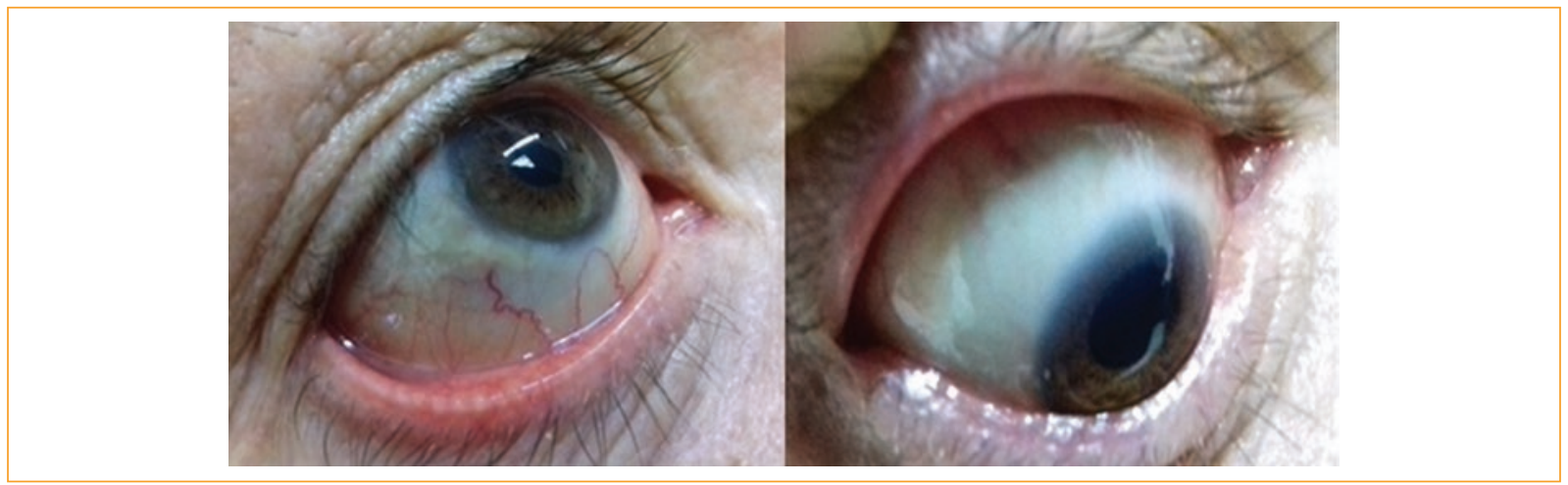

Figura 7. Paciente tratado con IRPL. En la imagen izquierda, obtenida después de la segunda sesión de tratamiento (día 15), se aprecia la presencia de menisco lagrimal de características normales. La imagen derecha, obtenida después de la tercera sesión de tratamiento (día 45), destaca la ausencia de hiperemia cilioconjuntival.

expresión del meibum ${ }^{16}$. Sin embargo, un mecanismo de este tipo solo induciría efectos a corto plazo ${ }^{17}$.

De acuerdo con el reconocido mecanismo de acción en el tratamiento de la piel en la rosácea sistémica ${ }^{18}$, es posible que la trombosis de la vasculatura que rodea las glándulas meibomianas pudiera desempeñar un papel en la disminución de la liberación local de mediadores inflamatorios. Además, la carga bacteriana en el margen palpebral y en los anexos oculares podría verse afectada directamente por la IRPL ${ }^{19}$.

Todos estos mecanismos y/o hipótesis producen un aumento significativo de la cantidad de lípidos en la superficie de la película lagrimal después del tratamiento, lo que sugiere que el flujo de meibum de las glándulas ha sido facilitado por el tratamiento con IRPL. Los beneficios, además, parecen ser acumulativos, de tal manera que, después de un curso de tres tratamientos durante 45 días, se reportan mejorías en los ojos tratados ${ }^{3-5}$.

Existe otra posibilidad de que la IRPL tenga el potencial de modificar la producción mitocondrial de especies oxidativas reactivas, que han estado implicadas en el ojo seco $20-23$.

Han sido documentados otros efectos de la IRPL como: fotomodulación ${ }^{24}$, activación de fibroblastos y síntesis de colágeno ${ }^{25,26}$, coagulación y necrosis del Demodex ${ }^{27,28}$, modulación y secreción de moléculas antinflamatorias ${ }^{29,30}$, entre otros.

Sería importante para estudios futuros realizar pruebas de eficacia del tratamiento, comparar con otros grupos de 
pacientes, incluir otras medidas objetivas como la osmolaridad de la lágrima. Además, realizar estudios a largo plazo, fuera del rango de las tres sesiones de tratamiento.

\section{Conclusiones}

- La aplicación de IRPL posibilitó un incremento del BUT en la mayoría de los pacientes, por encima de $10 \mathrm{~s}$, y se obtuvo relación significativa entre el procedimiento y el incremento de esta variable.

- Se constató, posterior al procedimiento, una disminución en la frecuencia de las características del borde palpebral, relacionadas con la DGM, así como una menor necesidad de presión aplicada para la expresión glandular en la mayoría de los pacientes.

- En más de la mitad de la muestra se obtuvo expresión glandular de líquido claro transparente, con un incremento significativo de pacientes en este grupo, posterior a la IRPL.

- La realización del procedimiento posibilitó una mejoría de los pacientes en lo referente a: síntomas de ojo seco, posibilidades de realización de actividades cotidianas y manejo de situaciones ambientales desfavorables.

- Los resultados presentados muestran una mejora tanto en los signos clínicos y síntomas del síndrome de ojo seco por DGM, después de un curso de tres sesiones de IRPL, durante un período de 45 días, no obstante, se requieren estudios futuros a largo plazo relacionados con este tratamiento.

\section{Responsabilidades éticas}

Protección de personas y animales. Los autores declaran que para esta investigación no se han realizado experimentos en seres humanos ni en animales.

Confidencialidad de los datos. Los autores declaran que han seguido los protocolos de su centro de trabajo sobre la publicación de datos de pacientes.

Derecho a la privacidad y consentimiento informado. Los autores declaran que en este artículo no aparecen datos de pacientes.

\section{Conflicto de intereses}

Los autores declaran que no existen conflictos de intereses.

\section{Financiación}

Los autores declaran que no han recibido financiación para la realización de la investigación.

\section{Bibliografía}

1. Lemp MA, Crews LA, Bron AJ, Foulks GN, Sullivan BD. Distribution of aqueous-deficient and evaporative dry eye in a clinic-based patient cohort. Cornea. 2012;31(5):472-78.

2. Qiao J, Yan X. Emerging treatment options for meibomian gland dysfunction. Clin Ophthalmol. 2013;7:1797-803.

3. Toyos R, Buffa CM, Youngerman S. Case report: Dry-eye symptoms improve with intense pulsed light treatment. Eye World News Magazine. 2005.

4. Toyos R, McGill W, Briscoe D. Intense pulsed light treatment for dry eye disease due to meibomian gland dysfunction: a 3-year retrospective study. Photomed Laser Surg. 2015;33(1):41-6.

5. Craig JP, Chen YH, Turnbull PR. Prospective trial of intense pulsed Light for the treatment of meibomian gland dysfunction. Invest Ophthalmol Vis Sci. 2015;56(3):1965-70.

6. Vegunta S, Patel D, Shen JF. Combination therapy of intense pulsed light therapy and meibomian gland expression (IPL/MGX) can improve dry eye symptoms and meibomian gland function in patients with refractory dry eye: a retrospective analysis. Cornea. 2016;35(3):318-22.

7. Ding J, Sullivan DA. Aging and dry eye disease. Exp Gerontol. 2012; 47(7):483-90.

8. Obata H. Anatomy and histopathology of human meibomian gland. Cornea. 2002;21:S70-4.

9. Borchman D, Foulks GN, Yappert MC, Milliner SE. Differences in human meibum lipid composition with meibomian gland dysfunction using NMR and principal component analysis. Invest Ophthalmol Vis Sci. 2012;53:337-47.

10. Graham JE, Moore JE, Jiru X, Moore JE, Goodall EA, Dooley JS, et al. Ocular pathogen or commensal: a PCR-based study of surface bacterial flora in normal and dry eyes. Invest Ophthalmol Vis Sci. 2007;48:5616-23.

11. Mathers WD, Shields WJ, Sachdev MS, Petroll WM, Jester JV. Meibomian gland dysfunction in chronic blepharitis. Cornea. 1991;10:277-85.

12. Knop E, Knop N, Millar T, Obata H, Sullivan DA. The International Workshop on Meibomian Gland Dysfunction: report of the subcommittee on anatomy, physiology, and pathophysiology of the meibomian gland. Invest Ophthalmol Vis Sci. 2011;52:1938-78

13. Arciniega JC, Wojtowicz JC, Mohamed EM, McCulley JP. Changes in the evaporation rate of tear film after digital expression of meibomian glands in patients with and without dry eye. Cornea. 2011;30:843-7.

14. Bilkhu PS, Naroo SA, Wolffsohn JS. Effect of a commercially available warm compress on eyelid temperature and tear film in healthy eyes. Optom Vis Sci. 2014;91:163-70.

15. Jiang $X, L v, H$ Song $H$, Zhang M, Liu $Y, H u X$, et al. Evaluation of the Safety and Effectiveness of Intense Pulsed Light in the Treatment of Meibomian Gland Dysfunction. J Ophthalmol. 2016;2016:1910694.

16. Nagymihályi A, Dikstein S, Tiffany J. The influence of eyelid temperature on the delivery of meibomian oil. Exp Eye Res. 2004;78(3):367-70.

17. Geerling G, Tauber J, Baudouin C. The international workshop on meibomian gland dysfunction: report of the subcommittee on management and treatment of meibomian gland dysfunction. Invest Ophthalmol Vis Sci. 2011;52(4):2050-64.

18. Schroeter CA. Haaf-Von Below S, Neumann HAM. Effective treatment of rosacea using intense pulsed light systems. Dermatol Surg. 2005;31:1285-9.

19. Farrell HP, Garvey M, Cormican M, Laffey JG, Rowan NJ. Investigation of critical inter-related factors affecting the efficacy of pulsed light for inactivating clinically relevant bacterial pathogens. J Appl Microbiol. 2010;108:1494-508.

20. Chung H, Dai T, Sharma S, Huang YY, Carroll J, Hamblin M. The nuts and bolts of low-level laser (light) therapy. Ann Biomed Eng. 2012;40:516-33.

21. Wakamatsu TH, Dogru M, Matsumoto $Y$. Evaluation of lipid oxidative stress status in Sjögren syndrome patients. Invest Ophthalmol Vis Sci. 2013;54:201-10.

22. Hamblin MR, Huang YY, Sharma SK, Carroll J. Biphasic dose response in low level light therapy-an update. Dose Response. 2011;9:602-18.

23. Hamblin MR, Demidova TN. Mechanisms of low level light therapy. Photobiological Sciences Online; 2008. Disponible en: http://www.photobiology.info/Hamblin.html. Último acceso 21 julio de 2014.

24. Farivar S, Malekshahabi T, Shiari R. Biological effects of low level laser therapy. J Lasers Med Sci. 2014;5(2):58-62.

25. Cuerda-Galindo E, Díaz-Gil G, Palomar-Gallego M, Linares-García Valdecasas $R$. Increased fibroblast proliferation and activity after applying intense pulsed light 800-1200 nm. Ann Anat. 2015;198:66-7.

26. Goldberg D. Current trends in intense pulsed light. J Clin Aesthet Dermatol. 2012;5(6):45-53.

27. Prieto V, Sadick N, Lloreta J, Nicholson J, Shea C. Effects of intense pulsed light on sun-damaged human skin, routine, and ultrastructural analysis. Lasers Surg Med. 2002;30(2):82-5.

28. Kirn T. Intense pulsed light eradicates Demodex mites. Skin Allergy News. 2002;33(1):37.

29. Lee S, Park K, Choi J. A prospective, randomized, placebo-controlled, double-blinded, and split-face clinical study on LED phototherapy for skin rejuvenation: clinical, profilometric, histologic, ultrastructural, and biochemical evaluations and comparison of three different treatment settings. $J$ Photochem Photobiol B. 2007;88(1):51-67.

30. Taylor M, Porter R, Gonzalez M. Intense pulsed light may improve inflammatory acne through TNF- $\alpha$ down-regulation. J Cosmet Laser Ther. 2014;16(2):96-103. 